\title{
ANALYSIS OF SOME INDICATORS OF TUBER- CULAR MORBIDITY IN VARNA REGION IN 2008
}

\author{
Gabriela Tsankova \\ Medical College - Varna, Bulgaria
}

\section{SUMMARY}

According to the information from the World Health Organization (WHO) about $1 \backslash 3$ of the world population i.e. 2 milliard people are infected with Mycobacterium tuberculosis and they are at risk that their condition may deteriorate.

More than 8 million people are annually infected and approximately 2 million die of tuberculosis.

Bulgaria is a country with an average level of incidence - between 12 to 80 per 100000 .

In Varna region the number of the people infected with tuberculosis in 2008 was $143-37$ patients more than those registered in 2007.

Aim: To make an analysis of some tubercular infection indicators in Varna region in 2008. Materials and methods: A research was carried out in the Pneumophthisiatric Clinic in Varna, concerning the patients undergoing treatment there in 2008. Apart from that information was collected from the Center for Disease Control at the Regional Sanitary Inspection in Varna. Results: The number of people infected with tuberculosis in 2008 was 143 - 37 more compared to 2007. The cases of pulmonary consumption had increased by 36 . There were patients infected with tuberculosis in Varna Municipality, Provadia, Dolni Chiflik, Aкsakovo, Vulchi dol, etc. The highest number of patients suffering from tuberculosis were among the people over 65 years of age and the unemployed between 30 and 35 years of age especially the ones with low standard of life. In the group of the infected people there were 17 children and the youngest patient was 9 months old. Susceptible to the disease were people with weak immune system, low social status, alcoholics, smokers and unemployed. Conclusion: Reducing the incidence of tuberculosis in Varna region can be achieved by: control over the range and quality of the specific immunoprophylaxis with BCG vaccine, raising the public health awareness of tuberculosis, enhancing and monitoring the activity of the non-governmental organizations (NGOs) that work on the problem of tuberculosis in the region of Varna.

Key words: tuberculosis, morbidity, risk factors

\section{INTRODUCTION}

Tuberculosis is a global problem with enormous medical and economic importance for all humans. According to the information from the World Health Organization (WHO) about $1 \backslash 3$ of the world population i.e. 2 milliard people are infected with Mycobacterium tuberculosis and they are at risk that their condition may deteriorate.

More than 8 million people are annually infected and approximately 2 million die of tuberculosis.

Bulgaria is a country with an average level of incidence - between 12 to 80 per 100000 .

In Varna region the number of the people infected with tuberculosis in 2008 was $143-37$ patients more than those registered in 2007.

\section{AIMS}

The purpose of the present research is to analyze some indicators of tubercular infection in Varna region in 2008.

\section{MATERIALS AND METHODS}

In Varna region, in 2008 the infected people and those under treatment were investigated. The information is received from The Municipality Pneumo-phthisiatric Clinic Ltd., Varna as well as from the Center for Disease Control at the Regional Sanitary Inspection in Varna .

\section{RESULTS AND DISCUSSIONS}

The number of people suffering from tuberculosis is 143. 134 of these cases present with pulmonary consumption There has been available evidence of the growth in both of the general number of cases of tuberculosis in 2008 and those of pulmonary consumption (with almost $1 \backslash 4$ ) in comparison with 2007. 


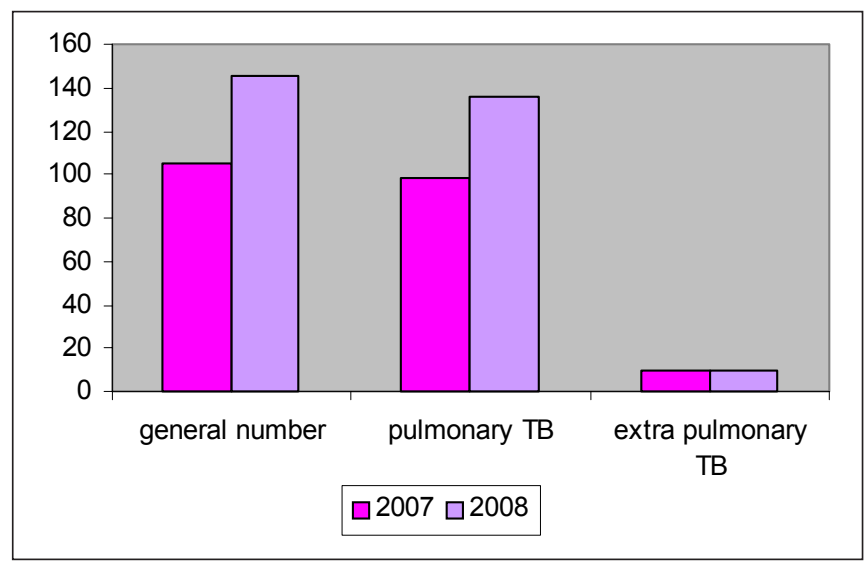

Fig. 1. General number of cases of tuberculosis, pulmonary and extra pulmonary TB in 2008 in comparison with 2007year.

Most of the tubercular cases are in the municipalities of Provadia - 9, Dolni Chiflik - 6 and Aksakovo - 5.

Figure 2 shows the distribution of the patients infected with tuberculosis according to their age. In 2008 the highest morbidity is registered-between 55 and $59 \mathrm{r-}$ $49.40 \%$ ooo ( 16 cases), between 40 and 44 years of age $49.10 \% 000$ ( 15 cases) and between 30-34 years of age $44.25 \%$ ooo (17 cases). The greatest number of the infected people are aged over 65 - 20 cases. Among those suffering from tuberculosis there are 17 children .In addition to this the youngest patient is nine months old.

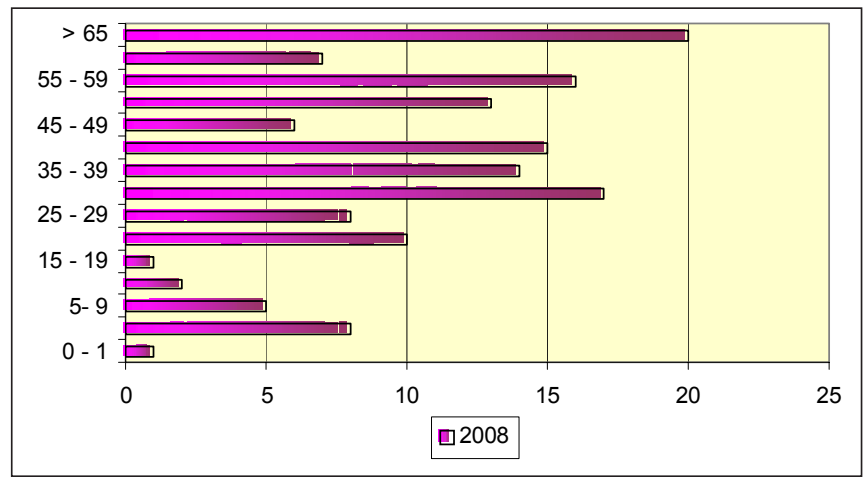

Fig. 2. The distribution of the people infected with tuberculosis according to their age

The distribution of the infected people in accordance with clinical form of tuberculosis has been shown in figures 3 and 4.

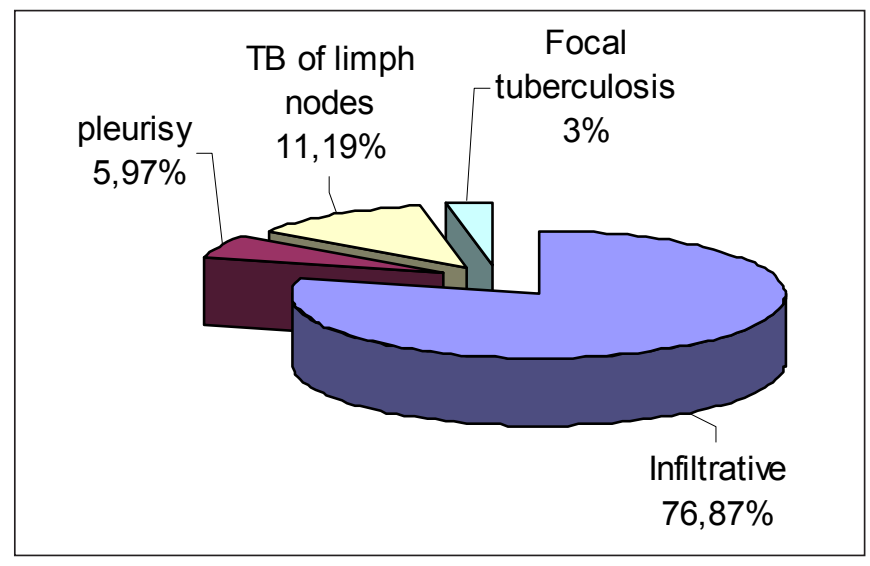

Fig. 3. The distribution of the people infected with tuberculosis according to the clinical forms

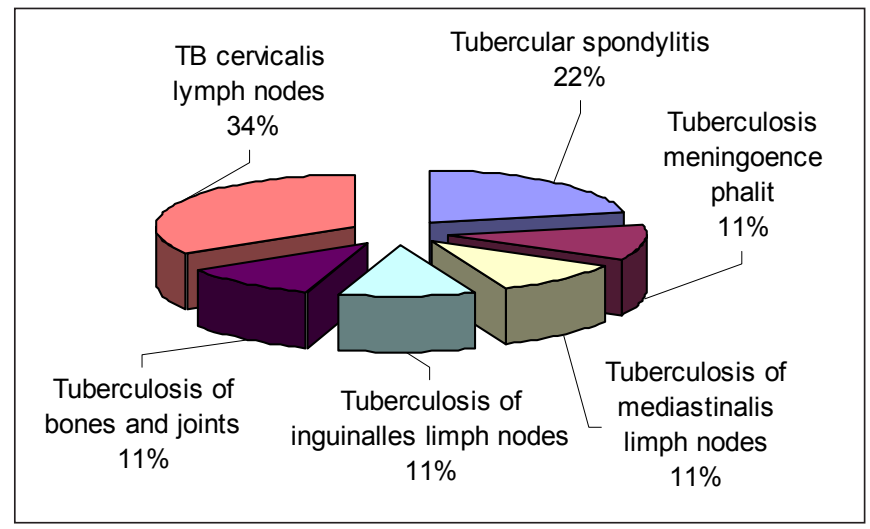

Fig. 4. Extrapulmonary forms of TB

Out of all 143 people suffering from TB, 134 are infected with pulmonary tuberculosis. Most of these people were infected with infiltrative pneumonia-103 (76.87\%) and tuberculosis of the lymph nodes -15 people $(11.19 \%)$. The most common form of extra pulmonary tuberculosis is the TB cervicalis lymph nodes.

An important indicator characterizing the tubercular epidemic is the bacillus secretion. The infected with tuberculosis bacillus secretors are 65 i.e. $45 \%$ of all affected by tuberculosis. (figure 5) 


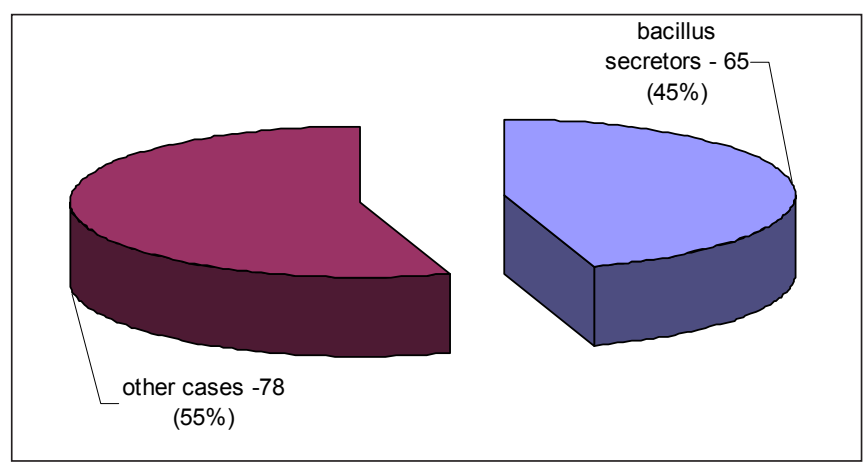

Fig. 5. Relative part from the bacillus secretors among the infected with tuberculosis in 2008.

Susceptibility to tuberculosis is increasing by the influence of a variety of risk factors which weaken the immune system - living conditions, inadequate nourishment, bad habits (drinking, smoking), former illness. In 2008 51$35.5 \%$ of the infected people are unemployed, while $37-25.87$ $\%$ of them have accompanying diseases: diabetes, heart diseases .From all suffering from TB there are 2 alcoholics and $58.74 \%$ of all examined patients are smokers. Smoking is generally regarded as a negative factor.
The number of the men infected with tuberculosis $(71 \%)$ is many times bigger than the number of the women $(18 \%)$. This fact is probably connected with the presence of more risk factors in their group. Children under 18 make up $18 \%$ of the people infected with tuberculosis.

\section{CONCLUSION.}

There has been evidence that proves the rise in the morbidity of tuberculosis and the increased number of bacillus secretors in Varna region in 2008. The growth in the number of the children infected with TB is alarming.

In relation to the collected data above, it is necessary to take the following measures:

1. The control over the range and quality of the specific immunoprophylaxis with BCG vaccine should be improved.

2. Timely diagnosis and treatment of the new patients, raising the public health awareness of tuberculosis particularly among the groups at risk should be ensured and promoted.

3. The actions of the non-governmental organizations (NGOs) that work on the problem of tuberculosis in the region of Varna should be monitored and stimulated.

\section{REFERENCES}

1. Bakardzhiev, L., D. Stephanova, V. Nichev.. Epidemiology of Tuberculosis and Chronic Non-specific Lung Diseases in the Republic of Bulgaria (1980-1992 г.). Pneumology and Phthisiatrics, C., XXVIII,
1991,3/4, 8-11.

2. Benchev, B. The tuberculosis as a global problem for public health. Infectious diseases, S., XXXVI, 2005, 1, 3-8. 3. Valkanova, N., Paunov, Tz., 2002, 2, 19-24.
Konstantinov, R., Some aspects of tubercular morbidity and drug resistance of M. tuberculosis strains in Varna region in period 1986-2000, Infectology, XXXIX,

Address for correspondence:

Gabriela Tsankova

Medical College-Varna

84, Tsar Osvoboditel Str.

9000 Varna, Bulgaria

e-mail: gabriela_sc@abv.bg 